\title{
Acceleration mechanism of radiation belt electrons through interaction with multi-subpacket chorus waves
}

\author{
Ryoko Hiraga ${ }^{*}$ (I) and Yoshiharu Omura
}

\begin{abstract}
We conduct test particle simulations to examine the acceleration mechanism of relativistic electrons through interaction with multi-subpacket chorus waves. As the analysis of recent observations reveals, amplitude of a rising tone element of chorus wave consists of many short wave packets. We call this single rising tone chorus element with the collective structure of multiple short wave packets as a multi-subpacket chorus wave. In this simulation, we develop the wave model with rapidly fluctuating amplitude and phase discontinuities across each subpacket, in order to examine how these features of multi-subpacket chorus wave influence the nonlinear trapping processes in efficient acceleration of relativistic electrons such as relativistic turning acceleration (RTA) and ultra-relativistic acceleration (URA). To conduct comprehensive examinations, we test more than nine million particles with various initial conditions covering the energy range from 100 to $6 \mathrm{MeV}$, and the equatorial pitch angles from $10^{\circ}$ to $89^{\circ}$. The test particles interact with a single rising tone element of multi-subpacket chorus wave set up with the maximum amplitude of about $2 \mathrm{nT}$ and the frequency rise from about $1.3 \mathrm{kHz}$ to $3.8 \mathrm{kHz}$ over $0.25 \mathrm{~s}$. Relativistic electrons are accelerated by about $160 \mathrm{keV}$ under preferable conditions. The energy increase verifies the high efficiency of acceleration by the wave-particle interactions, based on the fact that it is achieved by a short time interaction less than $1 \mathrm{~s}$ with a single element of chorus wave. By analyzing the detailed behavior of the accelerated electrons, we find successive trapping of the resonant electrons resulting in the efficient accelerations from the consecutive multiple subpackets of a chorus wave element.
\end{abstract}

Keywords: Wave-particle interaction, Test particle simulation, Particle acceleration, Relativistic electrons, Radiation belts, Whistler-mode chorus

\section{Introduction}

Prompt recovery of highly energetic $(\mathrm{MeV})$ electron populations in Earth's outer radiation belt, the outermost of the twin torus-shaped rings of high-energy plasma surrounding Earth, has been observed during geomagnetic storms (e.g., Foster et al. 2017). The prompt recovery includes efficient acceleration of radiation belt electrons though interaction with chorus waves such as relativistic turning acceleration (RTA) and ultra-relativistic acceleration (URA) (Omura et al. 2007; Summers and Omura

\footnotetext{
*Correspondence: ryoko.hiraga@gmail.com

Research Institute for Sustainable Humanosphere, Kyoto University, Kyoto, Japan
}

2007). Hence the wave-particle interaction and the consequent acceleration of electrons have been a topic of interest (e.g., Baker et al. 2018) concerning the formation of the outer radiation belt in Earth's magnetosphere.

As observed outside the plasmapause (Carpenter 1963) in Earth's inner magnetosphere by spacecraft and on the ground (e.g., Golkowski et al. 2019; Hosseini et al. 2019; Demekhov et al. 2017; Hosseini et al. 2017; Li et al. 2011; Meredith et al. 2003), chorus wave is a rising tone electromagnetic emission which is generated by an instability driven by the temperature anisotropy of energetic electrons (e.g., $10-100 \mathrm{keV}$ ) and propagates nearly parallel to Earth's magnetic field $\mathrm{B}_{0}$. When energetic electrons with the energy range of $10 \mathrm{keV}$ to $10 \mathrm{MeV}$ interact with 
chorus wave at the resonance velocity $V_{R}$, some of them fall into the wave potential, which is formed by Lorentz force of electron velocities perpendicular to Earth's dipole magnetic field $v_{\perp}$ and the wave magnetic field. These electrons continue to remain in resonance being phasetrapped in the potential, resulting in efficient acceleration such as RTA and URA. This indicates that through the intermediary of chorus waves excited by resonant lowerenergy electrons, energy is transferred from lower-energy electron population to the $\mathrm{MeV}$ radiation belt electrons.

In the acceleration process, variation of the wave potential during the wave-particle interaction is a key factor for the electrons to be trapped and accelerated by the chorus waves. The wave potential significantly depends on the wave amplitude: larger wave amplitude results in expansion of the wave potential, and hence efficient electron acceleration is more likely to occur (Santolík et al. 2014; Foster et al. 2017; Omura et al. 2019). An analysis of recent observation data by Van Allen probes (Santolík et al. 2014; Foster et al. 2017) shows a rising tone chorus element consists of many short wave packets with time scale of $10-30 \mathrm{~ms}$. We call this single rising tone chorus element with the collective structure of multiple short waves as multi-subpacket chorus wave. Besides a rapid amplitude variation, another feature of multi-subpacket chorus wave is to have phase discontinuities across each subpacket (Shoji and Omura 2013). Phase discontinuity here indicates that the chorus wave phase relative to the electron gyrophase is discontinuously changed when an electron in resonance with a chorus subpacket encounters the next subpacket. In this study, we conduct simulations of wave-particle interaction including the multi-subpacket wave structure and the phase discontinuities, and examine the efficiency of the electron acceleration processes (RTA and URA) under these conditions.

The simulation model of multi-subpacket chorus waves and conditions of relativistic electrons tested in the simulations are described in "Simulation method and wave model" section. In "Mechanism of efficient acceleration of relativistic electrons" section, to examine the acceleration mechanism, we analyze temporal variation of trajectory, energy and velocity of several representative electrons. Besides the representative electrons, it is also examined in "Comparison under different initial conditions" section if the acceleration mechanism discussed in "Mechanism of efficient acceleration of relativistic electrons" section is applicable to electrons under different conditions such as different energy levels. Finally, we summarize our simulation results and conclude this study in "Summary and conclusion" section.

\section{Simulation method and wave model Simulation model of multi-subpacket chorus wave elements}

In the present simulations, a pair of multi-subpacket chorus elements are generated on the equatorial plane (Santolík and Gurnett 2013) and propagate both northward and southward along $B_{0}$ as shown in Fig. 1 . The wave amplitude $B_{w}$ and frequency $\omega$ are based on the chorus equations, which describe time evolution of wave amplitude and frequency at the equator (Omura et al. 2009).

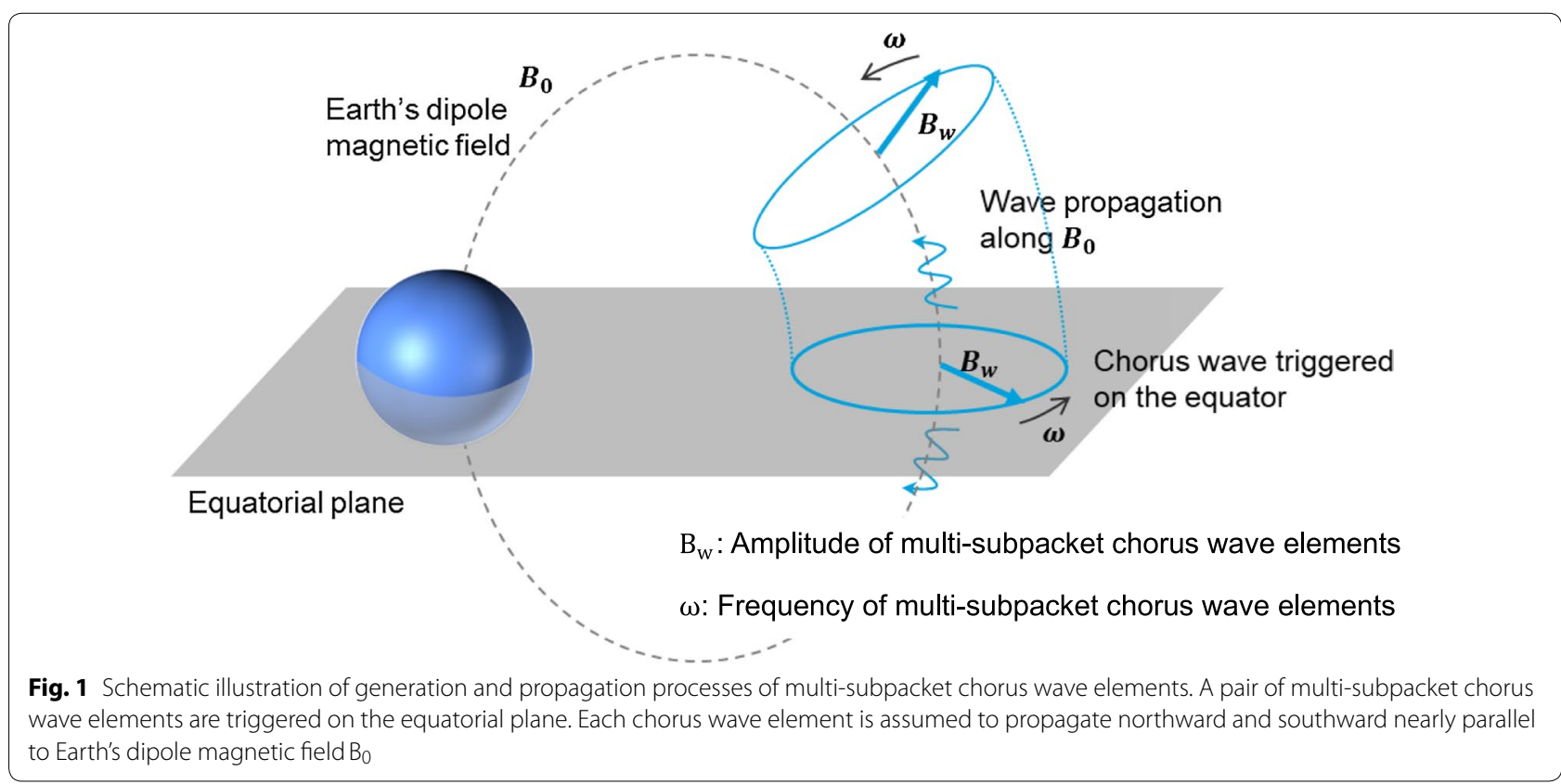


Their time variations on the equator are shown in Fig. 2a, b. The vertical axes of the figures are normalized by $\mathrm{B}_{0}$ and the equatorial cyclotron frequency $\Omega_{e 0}$. In our simulation we assume these variables as $270 \mathrm{nT}$ and $7.6 \mathrm{kHz}$, respectively, based on the recent observations (Omura et al. 2019). The horizontal time axes are also normalized by $\Omega_{e 0}$ in the figures. The figures, therefore, represent the amplitude variation with its maximum peak of about $2 \mathrm{nT}$ and the frequency increase from 1.3 to $3.8 \mathrm{kHz}$ over $0.25 \mathrm{~s}$ approximately.

Figure 3 shows the generation process of a multisubpacket chorus wave element in our simulation. Each subpacket composing the wave element is triggered by a group of seed electrons in the equatorial region and slightly upstream from the equator. The wave amplitude grows until it reaches the optimum amplitude (Omura and Nunn 2011) and starts decreasing down to the threshold level, which is the minimum value for subpackets to propagate. In our simulation, resonant seed electrons trigger a subpacket and are modulated by the subpacket during the interaction. The modulated electrons then trigger the next subpacket by cyclotron resonance when they are released by the previous subpacket. In Fig. 3, a group of resonant seed electrons which trigger packet 1 are accelerated through interaction with packet 1 and triggers packet 2 by cyclotron resonance instantly after the electrons are released by packet 1 . Multiple subpackets are generated continuously in this manner (Shoji and Omura 2013) to develop the wave amplitude model as shown in Fig. 2a. During the acceleration, the gyrophase of the seed electrons is modulated by the wave frequency. When the modulated seed electrons trigger the next subpacket, the frequency of the previous subpacket at the time of releasing the seed electrons is transferred to the next subpacket, which results in the gradual frequency increase as shown in Fig. 2b.

Another key assumption in this study is that a phase of each subpacket is randomized at each timing of packet generation in the simulation. Therefore, resonant electrons undergo phase discontinuities at every beginning of interaction with a new subpacket. As shown in Fig. 3, the phase difference between packet 2 and the interacting electrons $Z_{2}$, for example, are discontinuously changed to $Z_{3}$ when the electrons are released by packet 2 and start to interact with packet 3 .

\section{Simulations of wave-particle interaction for the test particles of comprehensive ranges of energy and equatorial pitch angle}

In the present simulations, the motion of relativistic electrons is derived by relativistic equations of motion (Omura and Summers 2006) under the environment of Earth's dipole magnetic field and multi-subpacket chorus waves propagating along the dipole field line. The time step and grid spacing are set as $\Delta t=0.05 \Omega_{e 0}^{-1}$ and $\Delta h=$ $0.05 \mathrm{c} / \Omega_{e 0}$, respectively, where $\Omega_{e 0}$ and speed of light $c$ are assumed as 1 . We create a system of $7500 c / \Omega_{e 0}$ for a hemisphere in the spatial dimension. For a comprehensive examination, the simulations are conducted for relativistic electrons under a wide range of initial conditions:

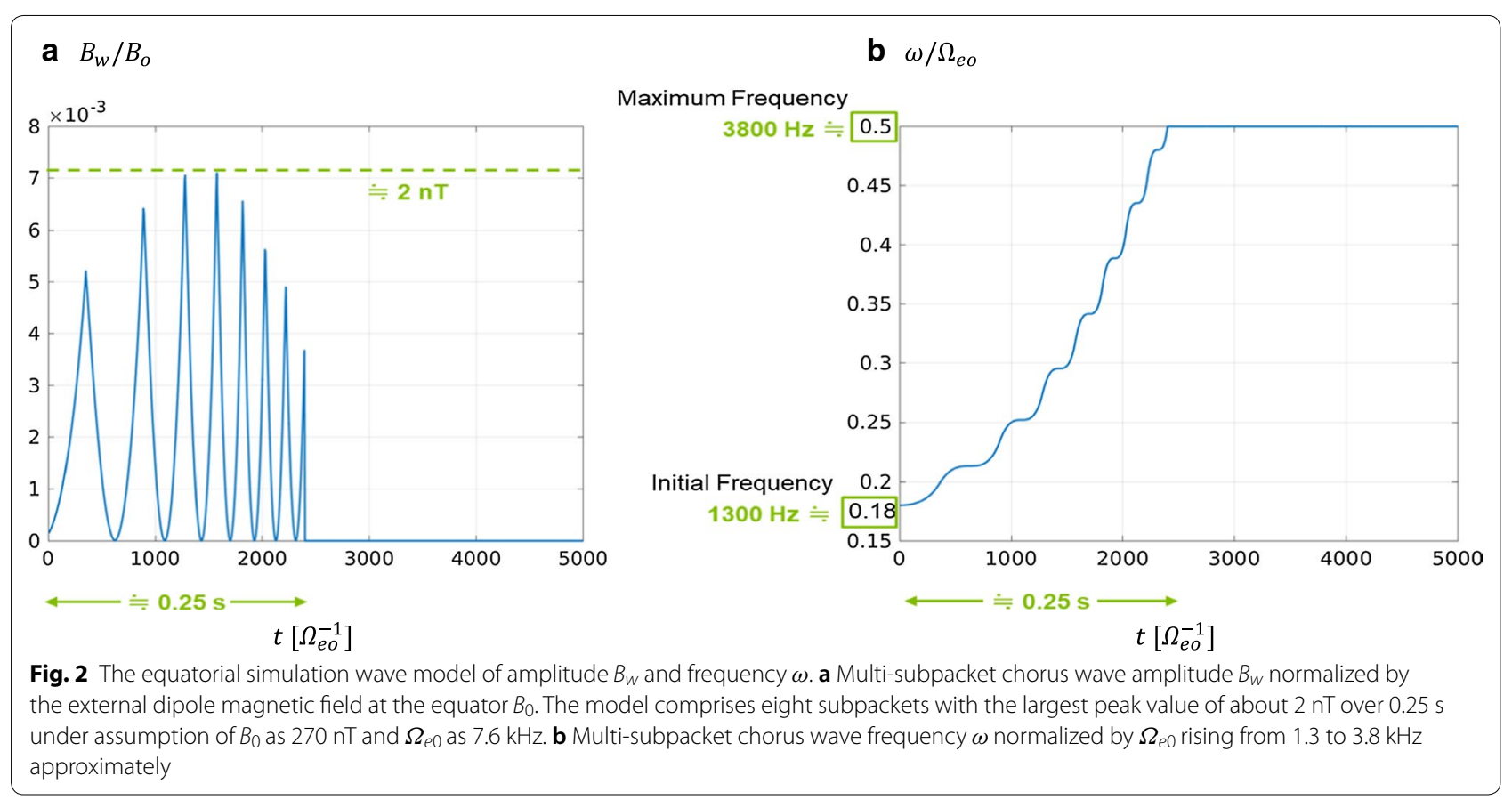




\section{- Optimum amplitude \\ - Threshold amplitude}
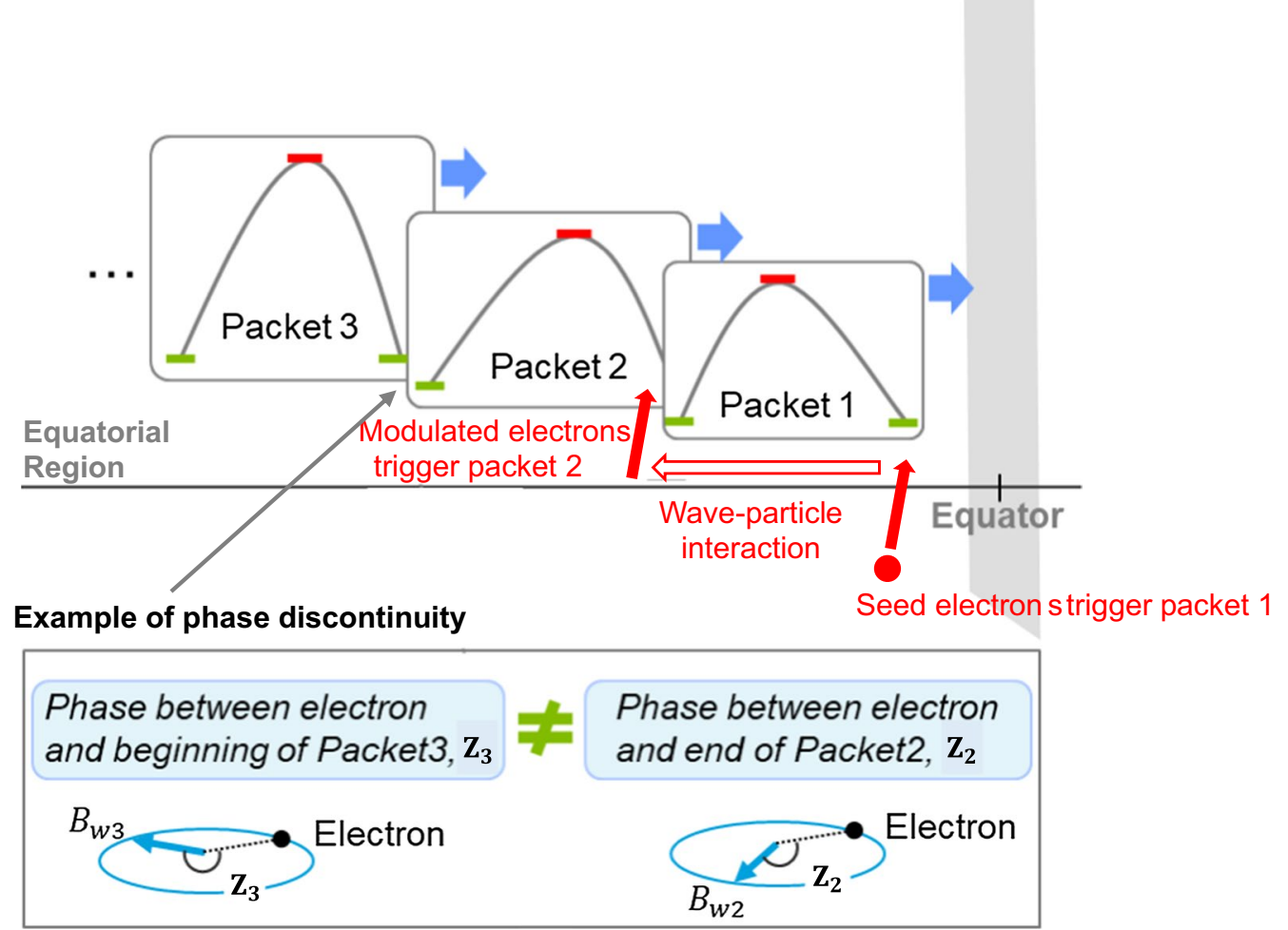

Fig. 3 Schematic diagram of generation process of subpackets in the equatorial region. Seed electrons generate a chorus wave subpacket (packet 1) by cyclotron resonance. The resonant seed electrons are modulated by packet 1 through the interaction. The frequency of packet 1 at the timing of releasing the seed electrons is transferred to packet 2 when the modulated seed electrons trigger packet 2 . Since phase of each subpacket is randomized in the simulation, phase difference between packet 2 and the resonant seed electrons gyrophase $Z_{2}$ is discontinuously changed to $Z_{3}$ at the timing of being released by packet 2 and interacting with packet 3

60 different initial energy levels from 100 to $6 \mathrm{MeV}$ and 43 cases for the initial equatorial pitch angles from $10^{\circ}$ to $89^{\circ}$. For each set of the initial energy and the equatorial pitch angle, 360 electrons with different gyrophases (the angle between electron velocity perpendicular to Earth's dipole magnetic field $\mathrm{v}_{\perp}$ and wave magnetic field $\mathrm{B}_{\mathrm{w}}$ ) shifted by $1^{\circ}$ are set up for 10 different initial locations evenly distributed over the distance between the equator and the mirror point on the northern hemisphere.

Figure 4 is an overview of energy increase by waveparticle interactions. An element of multi-subpacket chorus wave interacts with a group of electrons under each set of the initial conditions. We select the most successfully accelerated electron, which results in the largest energy increase out of 3600 electrons under each set of initial conditions and visualize the energy increase of the most successful electron in Fig. 4. As the figure shows, relativistic electrons under some conditions are accelerated by more than $100 \mathrm{keV}$. Such energy increase suggests the high efficiency of accelerations by the wave-particle interaction, since the interactions are with a single element of multi-subpacket chorus waves over a short period less than $1 \mathrm{~s}$. For detailed examination of this efficient acceleration process, several sample cases which resulted in a large energy increase (case 1, 2 and 3 in the figure) are featured in a later section.

\section{Mechanism of efficient acceleration of relativistic electrons}

In the examination of the acceleration process through wave-particle interaction, behavior of the wave potential becomes a key factor. What we define as the wave potential is the boundary condition and forms the trapping region, where electrons are entrapped by the chorus wave. Since the wave potential and the trapping region are significantly influenced by the magnitude of chorus wave amplitude, the structure of multi-subpacket wave amplitude makes the trapping region expand and shrink 


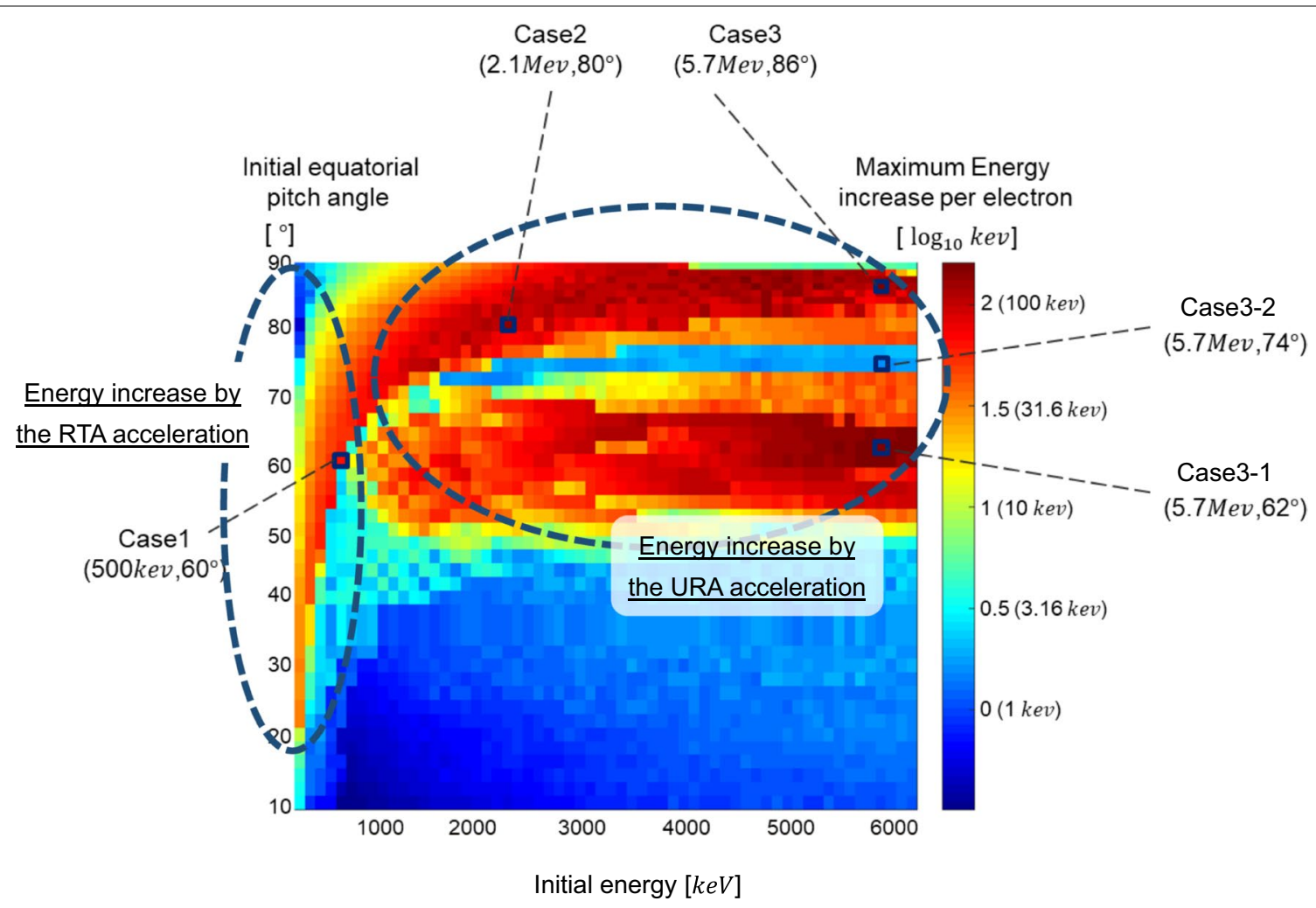

Fig. 4 Energy increase profile of the most successfully accelerated electron under each initial condition. Comprehensive simulations are conducted under various initial conditions: 60 energy levels and 43 equatorial pitch angles. Each condition is applied for 3600 electrons with different gyrophases and initial positions. The energy increase of the most successfully accelerated electron out of the 3600 electrons in each case is visualized in the colormap. The high energy increase range (colored in red) with lower initial energy electrons results from the RTA acceleration, whereas the range over MeV-class initial energy is due to the URA acceleration. The URA high energy range consists of two bands separated by a cavity at the initial pitch angle of around $70^{\circ}$. The upper band and lower band are formed by the different types of URA: overtaken URA and catching up URA, respectively. The cavity is the intermediate condition, where no efficient acceleration occurs

rapidly. It clearly affects the trapping process of relativistic electrons. In this section, we discuss the behavior of the trapping region during the wave-particle interaction.

\section{Theoretical background}

Temporal and spatial variation of $V_{R}$ are discussed in this section. $V_{R}$ is given by:

$$
V_{R}=\left(\omega-\Omega_{e} / \gamma\right) / k
$$

where $k$ is a wave number of a chorus wave, and $\Omega_{e}$ and $\gamma$ are gyrofrequency and Lorentz factor of an electron, respectively, given by $\Omega_{e}=\Omega_{e 0}\left(1+a h^{2}\right)$, where $a$ is a curvature of Earth's dipole magnetic field, and $\gamma=\left[1-\left(V_{R}^{2}+v_{\perp}^{2}\right) / c^{2}\right]^{-1 / 2}$. By substituting $\gamma$ as a function of $V_{R}$ in Eq. (1), $V_{R}$ can be explicitly obtained as (Summers et al. 2012):

$$
\frac{V_{R}}{c}=\frac{\frac{1}{\chi \xi}\left(\frac{\omega}{\Omega_{e}}\right)^{2}-\left[\frac{1}{\chi^{2} \xi^{2}}\left(\frac{\omega}{\Omega_{e}}\right)^{4}+\left\{\frac{1}{\chi^{2} \xi^{2}}\left(\frac{\omega}{\Omega_{e}}\right)^{2}+1\right\}\left\{1-\left(\frac{\omega}{\Omega_{e}}\right)^{2}-\left(\frac{v_{\perp}}{c}\right)^{2}\right\}\right]^{1 / 2}}{\left[\frac{1}{\chi^{2} \xi^{2}}\left(\frac{\omega}{\Omega_{e}}\right)^{2}+1\right]}
$$


where $\xi$ and $\chi$ are dimensionless parameters given by $\xi^{2}=\omega\left(\Omega_{e}-\omega\right) / \omega\left(\Omega_{e}-\omega\right) \omega_{\mathrm{pe}}^{2} \quad$ including electron plasma frequency $\omega_{\mathrm{pe}}$, and $\chi^{2}=1 /\left(1+\xi^{2}\right)$. Since $V_{R}$ is a function of distance from the equator $h$ and time $t$, the resonance velocity $V_{R}$ takes different values instantaneously at each location and timing of the wave-particle interaction.

\section{Trapping region in the velocity phase space}

The condition for electrons to be entrapped by chorus wave is described by the second-order nonlinear ordinary differential equation (Dysthe 1971; Nunn 1974; Matsumoto and Omura 1981; Omura et al. 2008, 2009):

$$
\frac{d^{2} \zeta}{\mathrm{dt}^{2}}=\frac{\omega_{t}^{2} \chi^{2}}{\gamma}(\sin \zeta+S)=\omega_{\mathrm{tr}}^{2}(\sin \zeta+S),
$$

where $\omega_{\mathrm{tr}}=\chi \sqrt{k V_{\perp 0} \Omega_{w} / \gamma}$, and $V_{\perp 0}$ is the average perpendicular velocity of energetic electrons. The parameter $S$ is an inhomogeneity factor given by:

$$
S=-\frac{1}{s_{0} \omega \Omega_{w}}\left(s_{1} \frac{\partial \omega}{\partial t}+\operatorname{cs}_{2} \frac{\partial \Omega_{e}}{\partial h}\right),
$$

where

$$
s_{0}=\frac{\chi}{\xi} \frac{V_{\perp 0}}{c},
$$

$$
\frac{\mathrm{dK}}{\mathrm{dt}}=\frac{\mathrm{eB}_{w}}{k} v_{\perp} \sin \zeta,
$$

where $K$ is energy of the electron. The integral of the sinusoidal term over $0 \leq \zeta \leq 2 \pi$ gives no energy to

where $V_{P}$ is the phase velocity of the chorus wave given by $V_{P}=c \chi \xi$. To interpret the trajectory of $\zeta$ in Eq. (3) visually, we describe it in quadratic form by introducing a variable $\theta=\mathrm{d} \zeta / \mathrm{dt}$. By substituting $\theta$ to Eq. (3) and taking integration, we obtain:

$$
\theta^{2}+2 \omega_{\text {tr }}^{2}(\cos \zeta-S \zeta)=C
$$

where $C$ is an integral constant.

Figure 5 shows trajectories of Eq. (8) in the $(\theta-\zeta)$ space for various values of $C$ with a fixed value of $S=-0.4$. In the figure, some trajectories oscillate over $0 \leq \zeta \leq 2 \pi$ (such as the blue open line), whereas some of them draw closed lines oscillating within a limited range of $\zeta$ (such as the red closed trajectory). The variation of the kinetic energy is given by (Omura et al. 2007): . . 
electrons on the open trajectories, whereas those on the closed trajectories continue to gain energy until they get off the loop. When electrons are in the closed trajectory region, the electrons are interpreted as being entrapped by chorus wave, and the region is called trapping region (white colored region). We define the trajectory which forms the boundary of the trapping region as separatrix. The integration constant of separatrix $\left.C\right|_{\theta=0, \zeta=\zeta_{1}}$ can be obtained by Eq. (8) and the trajectory can be given by:

$$
\theta_{S}(\zeta)= \pm \omega_{\operatorname{tr}} \sqrt{2\left[\cos \zeta_{1}-\cos \zeta+S\left(\zeta-\zeta_{1}\right)\right]} .
$$

In this paper, we examine the trapping process of electrons in velocity phase space, and convert Eq. (10) by simply substituting $\theta=k \Delta v=k\left(v_{\|}-V_{R}\right)$ :

$$
v_{\| s}(\zeta)=\mathrm{V}_{\mathrm{R}} \pm v_{\alpha} .
$$

We have introduced a parameter $v_{\alpha}$ given by $v_{\alpha}=\frac{V_{\text {tr }}}{2} \sqrt{2\left[\cos \zeta_{1}-\cos \zeta+S\left(\zeta-\zeta_{1}\right)\right]}$, where trapping velocity $V_{\mathrm{tr}}=2 \omega_{\mathrm{tr}} / k$. Equation (11) indicates resonant electrons with $v_{\|}$inside the separatrix $\left(\left|v_{\|}-V_{R}\right|<v_{\alpha}\right)$ are entrapped by chorus wave.

\section{Successive trapping}

In this section, we discuss the mechanism of efficient energy increase of electrons by interaction with multisubpacket chorus waves by tracking the relation between the trapping region in velocity phase space and the actual velocity of electrons $v_{\|}$at each moment of the wave-particle interaction. Figure 6 shows the trajectory and energy variation of a sample electron with the initial energy of $500 \mathrm{keV}$ and equatorial pitch angle of $60^{\circ}$. Figure $6 \mathrm{a}$ shows the trajectory of the electron, which starts gyromotion from the equator (1. starting point) and travels northwards until it changes the traveling direction at the mirror point (2. mirror point). The colormap in the figure represents the profile of a pair of multi-subpacket chorus elements generated at the equator. One of the elements has a positive velocity and propagates northward from the equator, while the other propagates southward with a negative velocity. Before the electron reaches location $\mathrm{A}$ in the figure, it interacts with triggering waves (colored in dark blue in the profile) with a constant amplitude and frequency in the simulation. The amplitude is $1.5 \times 10^{-4}$ $\left[B_{e 0}\right]$ being slightly above the threshold value and the frequency is $0.18\left[\Omega_{e 0}\right]$. The triggering waves are set up for the entire system space initially, in order to enable the wave elements to propagate through the space. The electron begins to interact with subpackets of a chorus wave element at location $\mathrm{A}$ and finishes the interactions with all the eight subpackets at location B.
In Fig. 6b, the black line represents the variation of $V_{R}$. The value of $V_{R}$ in the figure decreases over the trajectory from the starting point to the mirror point due to increasing $\Omega_{e}$ as the electrons travel to higher latitude regions. When the electron interacts with the multisubpacket chorus wave between location $\mathrm{A}$ and $\mathrm{B}, V_{R}$ shows a rapid increase, as a result of the increasing wave frequency $\omega$. The yellow region around the trajectory of $V_{R}$ is the trapping region, which is enclosed by separatrix $v_{\| s}(\zeta)=V_{R} \pm v_{\alpha}$ from Eq. (11). The region is proportional to the square root of the wave amplitude $\Omega_{w}$ in the form of trapping velocity $v_{t r}=\chi \sqrt{\nu_{\perp} \Omega_{w} / k \gamma}$. In the figure it is clearly seen that the trapping region is affected by the distinctive wave amplitude structure of multi-subpacket chorus waves, expanding during the wave amplitude growth and shrinking during the wave amplitude drop. The blue line in Fig. $6 \mathrm{~b}$ draws the variation of $v_{\|}$, which starts the adiabatic gyromotion from the equator with a positive velocity of around $v_{\|}=0.4[c]$ northwards. When the sample electron interacts with a wave subpacket at location A, the velocity $v_{\|}$is located within the trapping region and the electron is entrapped by the chorus wave. The process can be seen by the trajectory of the $v_{\|}$being disturbed to follow the variation of $V_{R}$ in the figure.

When energetic electrons interact with a single subpacket of a multi-subpacket chorus element satisfying $\left|v_{\|}-V_{R}\right|<v_{\alpha}$, they fall into the trapping region. Some of the electrons get off the trapping region when it shrinks due to the wave amplitude drop, while some resonant electrons can remain in the shrinking region and continue to be accelerated until the end of the interaction. When the resonant electrons encounter the next wave subpacket, some of them again fall into the trapping region generated by the subpacket. In this manner electrons are entrapped and detrapped at some possibilities at each interaction with the consecutive subpackets of a chorus element. The sample electron shown in Fig. 6 is an example which encounters the first subpacket of a multisubpacket chorus element at location A, and falls in the trapping region. The electron remains in the trapping region generated by all the eight subpackets it passed through. As a result, the energy level shown in Fig. 6c continues to increase more than $60 \mathrm{keV}$ throughout the entire interaction with the chorus wave element. We define this trapping process by the consecutive multiple subpackets of chorus wave as successive trapping.

When an electron is under successive trapping, the angle between chorus wave and the electron $\zeta$ oscillates within a limited range such as $\zeta_{1}$ and $\zeta_{2}$ in Fig. 5. Figure 6d shows the variation of $\zeta$ against $\Delta v=v_{\|}-V_{R}$. When the electron is not in resonance with the multi-subpacket 


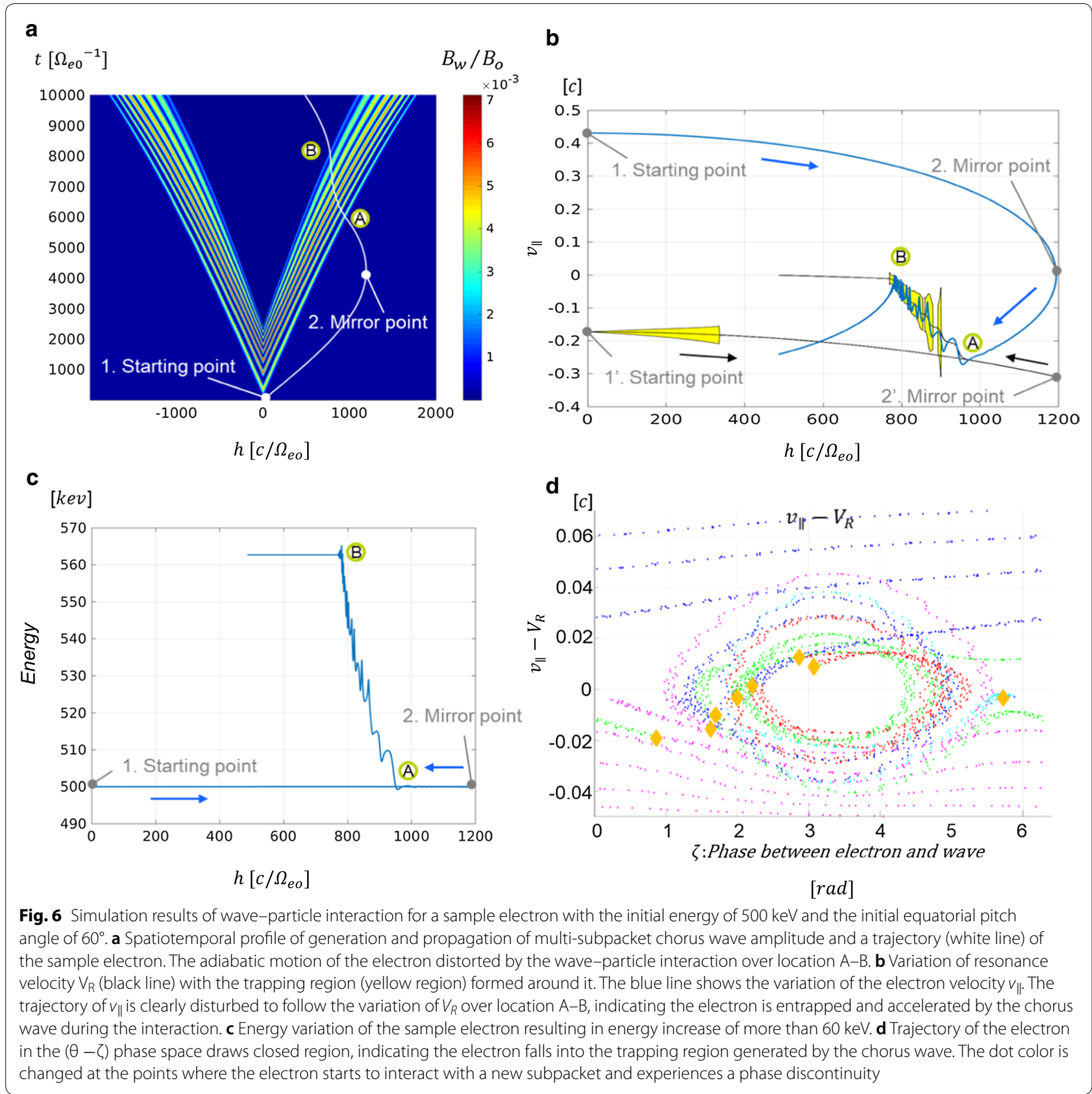

chorus wave outside the trapping region $\left(|\Delta v|>v_{\alpha}\right), \zeta$ rapidly oscillates between 0 and $2 \pi$. When $v_{\|}$falls into the trapping region $\left(|\Delta v|<v_{\alpha}\right)$, the $\zeta$ oscillation is limited within a certain range to draw a closed trajectory. Once the electron is detrapped, $\zeta$ is back to the rapid oscillation between 0 and $2 \pi$. Color of the dots in the figure is changed at each timing of the electron interacting with a new subpacket. In another word, the electron experiences phase discontinuities at each color change. This verifies that an electron can be entrapped by a series of multiple subpackets involving phase discontinuities.

\section{Comparison under different initial conditions} Successive trapping of relativistic electrons at different energy levels

In this section, occurrence of the successive trapping is examined under different initial conditions in addition to the case discussed in "Successive trapping" section. We introduce simulation results in three representative cases. Case 1 is the aforementioned case, in which the initial energy of test particles are set as $500 \mathrm{keV}$. In cases 2 and 3, the initial energies are set as $2.1 \mathrm{MeV}$ and $5.7 \mathrm{MeV}$, respectively. We include 3600 particles with the same 
initial energy and equatorial pitch angle in each case of simulation, and the most successful electron resulting in the maximum energy increase of the 3600 particles is shown in Fig. 7. Each column of the figure shows the temporal variations of key parameters such as electron's trajectory, energy increase and velocity $v_{\|}$in comparison with the trapping region.

As shown in case 1 described in "Successive trapping" section, the rows of $v_{\|}$in cases 2 and 3 show the occurrence of successive trapping by the variation of $v_{\|}$falling and remaining in the trapping region through the interaction with the entire rising tone element of multi-subpacket chorus wave. Energy increase can be seen in the raw of energy variation corresponding to the successive trapping processes in all the cases. The amount of the energy increases in cases 2 and 3, however, achieve more than double the result in case 1 . This is due to the manners of acceleration defined as relativistic turning acceleration (RTA) in Omura et al. (2007) and ultrarelativistic acceleration (URA) in Summers and Omura (2007). Electrons with low energy levels can get in resonance and entrapped when they interact with chorus wave traveling in the opposite direction to the wave propagation $\left(V_{R}<0\right)$. On the other hand, resonance and trapping for electrons with higher energy levels can occur when the electrons and chorus wave are moving in the same direction $\left(V_{R}>0\right)$, which enables a longer wave-particle interaction, and results in more efficient

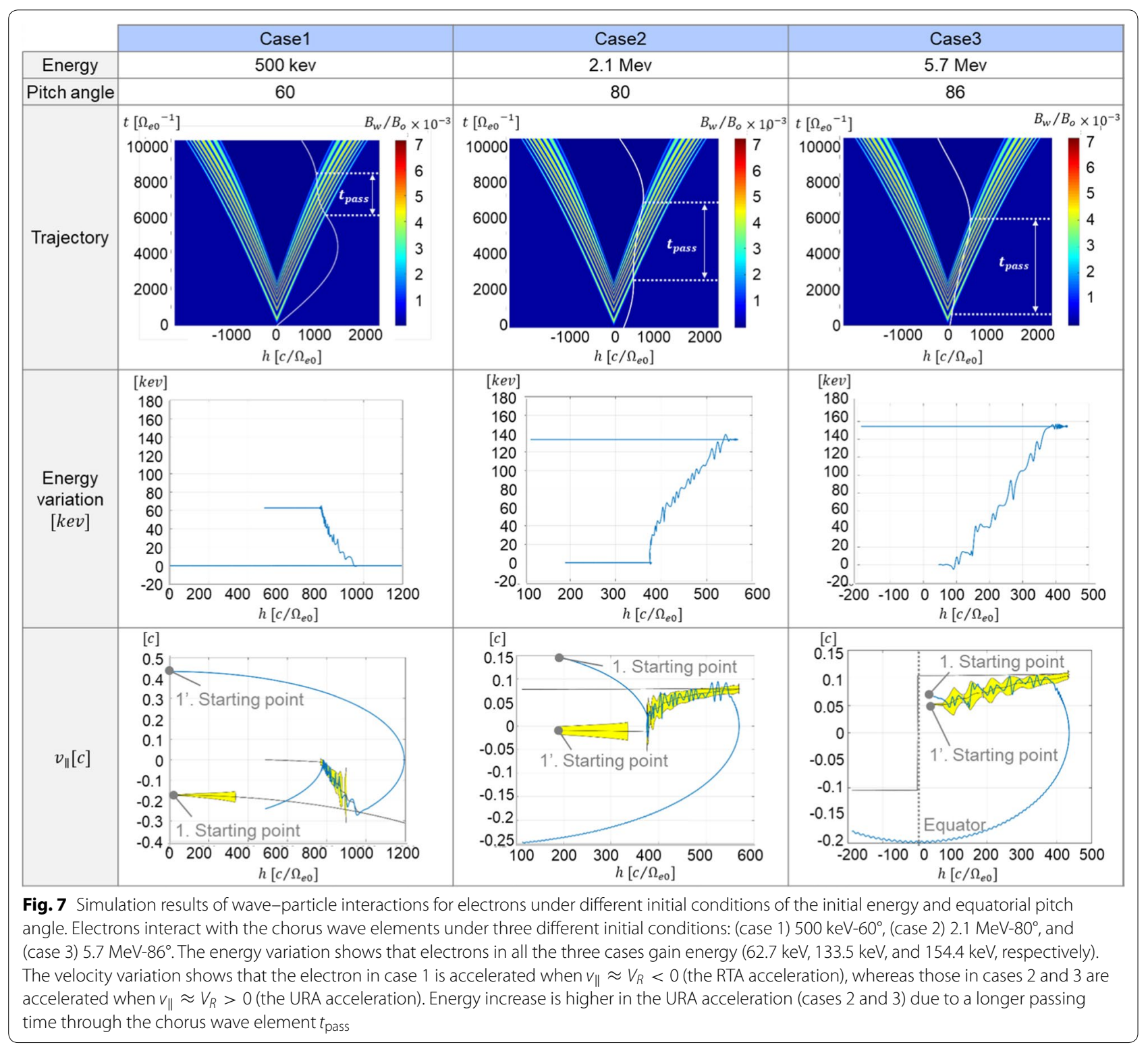


energy increase. In the rows of $v_{\|}$variations in Fig. 7, it can be seen that the electrons are entrapped at $V_{R}<0$ for case 1 and $V_{R}>0$ for the higher energy cases. Since the electrons in cases 2 and 3 are accelerated by the URA processes, they result in more efficient energy increase than those in case 1.

As a theoretical interpretation, the energy increase $\Delta K$ is given by:

$$
\Delta K \propto\left|E_{\perp}\right| v_{\perp} t_{\text {pass }}
$$

$t_{\text {pass }}$ is the time duration of electron passing the wave packets defined as (Hsieh and Omura 2017):

$$
t_{\text {pass }}=\frac{\lambda_{L}}{\Delta V}
$$

where $\lambda_{L}$ is the spatial length of the wave packet regarded as a constant value in this simulation since all cases use the same wave model and take the identical value of $\lambda_{\mathrm{L}}$. It is clear from the trajectories in Fig. 8 that passing time in case 1 (RTA) is shorter than those in cases 2 and 3 (URA). Since $\Delta K$ is proportionally affected by $t_{\text {pass }}$ in Eq. (12), the electron in case 1 results in less energy increase than those in cases 2 and 3.

\section{Overtaken URA and catching up URA}

Energy gain of the most successful electrons by successive trapping under different initial conditions (energy and equatorial pitch angle) is summarized in the color map previously shown in Fig. 4. The high energy increase range (colored in red) with lower initial energy electrons results from the RTA acceleration including case 1, whereas the range over $\mathrm{MeV}$-class initial energy is due to the URA acceleration such as cases 2 and 3 . In the figure, the URA high-energy range consists of two bands separated by a cavity at the initial pitch angle around $70^{\circ}$. In the two bands (upper and lower bands), the URA acceleration occurs in a different manner. We take case 3 as an example of the URA acceleration in the upper band. The trajectory of the most successful electron in case 3 is once again shown in Fig. 8a. The vectors in the figure, $\overline{V_{g}}$ and $\bar{v}_{\|}$, are velocity vectors of chorus wave group velocity and electron velocity. Both velocities are assumed as nearly parallel to $B_{0}$ in this study. As can be seen by the directions of the velocity vectors, the electron travels slower than the chorus wave propagation. The URA acceleration, therefore, occurs when the electron is overtaken by the chorus wave approaching from the behind. We define this manner of the URA acceleration as overtaken URA. As an example case in the lower URA band, we take the most successful electron with the same initial energy as case $3(5.7 \mathrm{MeV})$ but a lower equatorial pitch angle of $62^{\circ}$ (defined as case 3-1). The spatiotemporal trajectory of the electron is shown in Fig. 8b. As opposed to case 3 , the velocity vectors in this case indicate that the electron travels faster than the chorus wave propagation. As a result, the URA acceleration occurs when the electron catches up with the chorus wave element. We defined this type of URA as catching up URA in contrast to the overtaken URA in case 3.

There is always the intermediate case between the overtaken URA and the catching up URA, in which electrons travel at the velocity so close to the chorus wave propagation that no wave-particle interactions occur

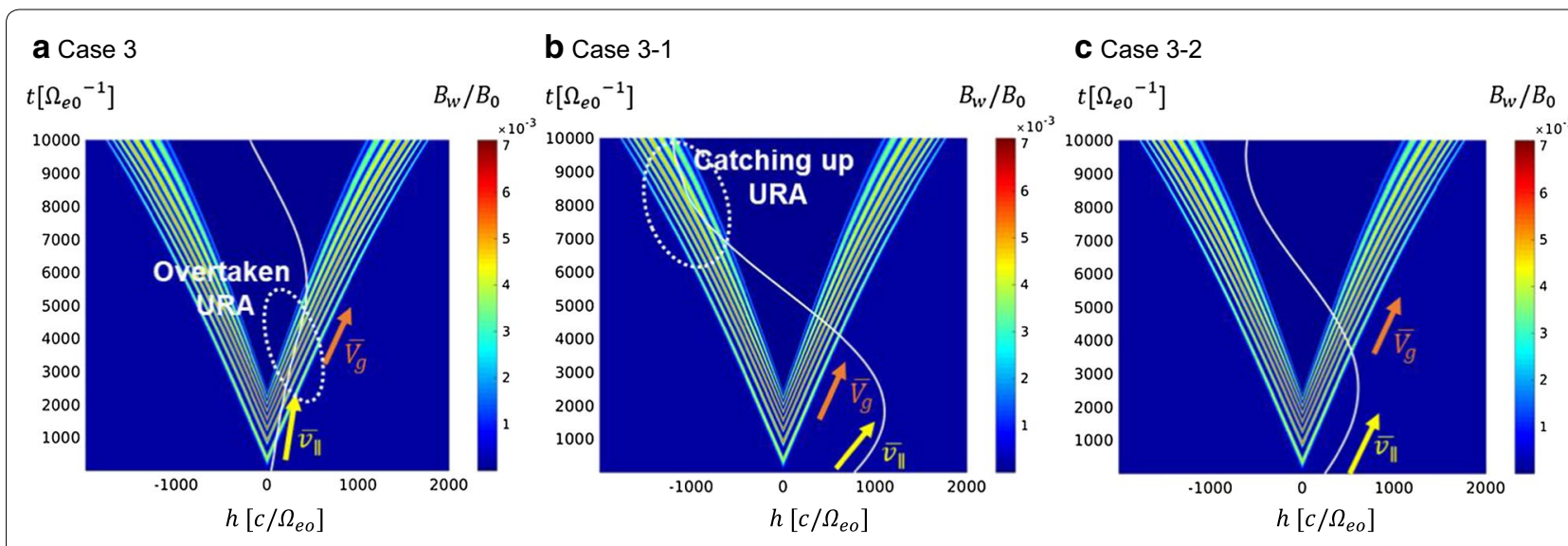

Fig. 8 Different types of the URA acceleration depending on the interrelation between $v_{\|}$and $V_{g}$. a Trajectory of the most successful electron in case $3\left(5.7 \mathrm{MeV}-86^{\circ}\right)$ is again taken as an example. The electron travels slower than the chorus wave propagation $\left(V_{\|}<V_{g}\right)$. The electron is accelerated by URA when it is overtaken by the chorus wave approaching from the behind (overtaken URA). b Trajectory in case 3-1 (5.7 MeV-62 ). The electron travels with the velocity $V_{\|}>V_{g}$, and is accelerated by URA when it catches up with the chorus wave going ahead (catching up URA). c Trajectory in case $3-2\left(5.7 \mathrm{MeV}-74^{\circ}\right)$. The electron travels with $v_{\|} \approx V_{g}$ and is neither overtaken nor catching up with the chorus wave, thus the efficient URA acceleration does not occur 
when electrons are traveling in the same direction as the wave propagation, resulting in no occurrence of the URA acceleration. This intermediate case can be seen as a cavity of the energy color map in Fig. 4. As an example case of the cavity region, we take case 3-2 with the intermediate equatorial pitch angle $\left(74^{\circ}\right)$. The trajectory of the most successful electron in case 3-2 is shown in Fig. 8c. It is clearly shown in the figure that the electron is neither overtaken nor catching up on the chorus wave element. In this study, the cavity appears for electrons with the initial equatorial pitch angle of around $70^{\circ}$. However, the condition depends on the interrelation between velocities of the traveling electrons and the chorus wave propagation. Therefore, the location of the cavity (the value of equatorial pitch angle) varies with parameters such as wave packet length.

\section{Probability of successive trapping}

In the previous sections, we have discussed the successive trapping in the RTA and URA acceleration processes by taking examples of the most successfully accelerated single particles. In this section, probabilities of occurrence of the successive trapping in the RTA and URA processes are examined: what fraction of particles besides the most successful particle undergo the successive trapping and are accelerated in what degree. Figure 9 shows the temporal energy variations of 360 particles equally distributed in gyrophase under the initial conditions of case $1\left(500 \mathrm{keV}-60^{\circ}\right)$, case $2\left(2.1 \mathrm{MeV}-80^{\circ}\right)$, and case 3 $\left(5.7 \mathrm{MeV}-86^{\circ}\right)$ as defined in Fig. 7. The top line reaching the largest energy increase at the termination of the simulation in each case represents the most successful particle discussed in Fig. 7. The most successful electron in case 1 , for example, has gained energy of $\Delta \mathrm{K}=62.7 \mathrm{keV}$ by the interactions with eight wave subpackets. Since the gradient of the energy increase is almost uniform over the interactions with the eight wave subpackets as shown in Fig. 9a, we assume the energy increase from each subpacket as $7.84 \mathrm{keV}$ by evenly dividing the final energy increase by the eight subpackets. Similarly Fig. 9b, c show case 2 and case 3, where the energy increases of the most successful electrons are achieved $\Delta \mathrm{K}=133.5 \mathrm{keV}$ and $154.4 \mathrm{keV}$, respectively, by interactions with seven wave subpackets. The energy gains per subpacket is assumed as $19.1 \mathrm{keV}$ and $22.1 \mathrm{keV}$. Figure 10 shows histograms of the final energy levels of the 360 test particles in cases 1, 2 and 3. From the histogram in Fig. 10a, a majority (about one-third) of the particles in case 1 are accelerated by around $20 \mathrm{keV}$, which is interpreted as energy gain from two to three subpackets based on the assumption that energy gain per subpacket is $7.84 \mathrm{keV}$. Rest of the particles also gain energies (less or more than $20 \mathrm{keV}$ ) depending on the number of the wave subpackets that each particle is entrapped by. The distribution of the histogram indicates that not only a small fraction of successful particles, but also many other particles can experience successive trapping by several subpackets. This is also true for cases 2 and 3 as shown in Fig. 10b, c.

Figure 11 shows the transition of the number of electrons being in resonance with the multi-subpacket
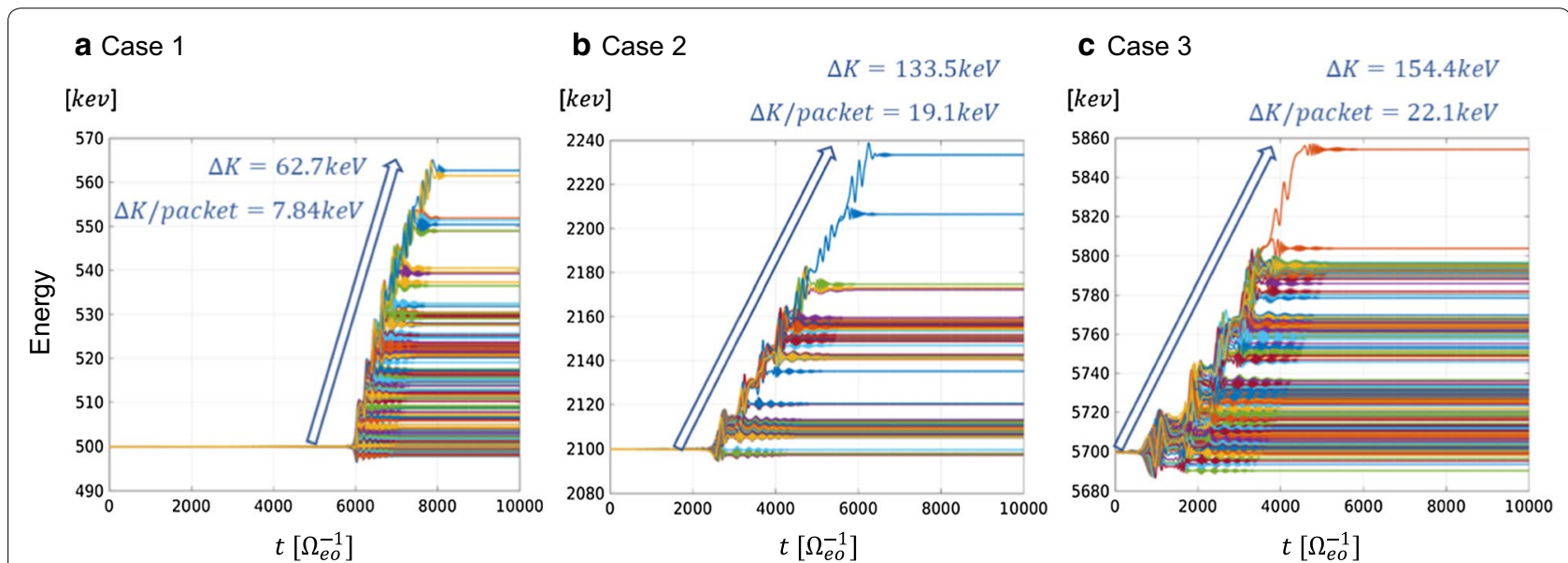

Fig. 9 Temporal energy variations of 360 particles under the initial conditions of case $1\left(500 \mathrm{keV}-60^{\circ}\right)$, case $2\left(2.1 \mathrm{MeV}-80^{\circ}\right)$, and case $3\left(5.7 \mathrm{MeV}-86^{\circ}\right)$. The energy variations of the 360 particles in three cases by the interactions with the chorus wave. The top line reaching the largest energy increase at the termination of the simulation in each case represents the most successful particle discussed in Fig. 7. a The most successful electron in case 1 gained energy of $\Delta K=62.7 \mathrm{keV}$ by the interactions with eight wave subpackets. The energy increase per subpacket, therefore, is $7.84 \mathrm{keV}$ assuming a uniform gradient of the energy increase. $\mathbf{b}$ The energy increase of the most successful electron in case 2 is 133.5 keV through interactions with seven subpackets, hence the energy gain per subpacket is calculated as $19.1 \mathrm{keV}$. c Similarly, the energy increase of the most successful electron in case 3 is $154.4 \mathrm{keV}$ through interactions with seven subpackets and the energy gain per subpacket is $22.1 \mathrm{keV}$ 


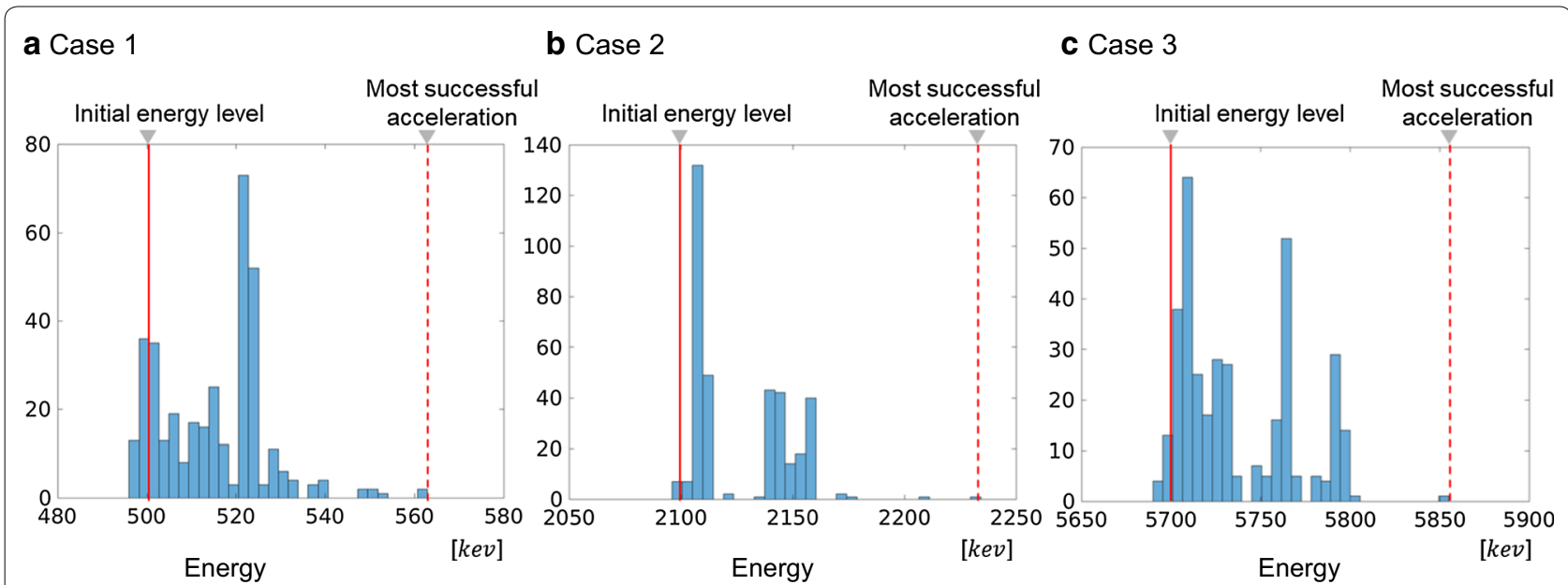

Fig. 10 Histograms of the final energy levels of 360 test particles in case $1\left(500 \mathrm{keV}-60^{\circ}\right)$, case $2\left(2.1 \mathrm{MeV}-80^{\circ}\right)$, and case $3\left(5.7 \mathrm{MeV}-86^{\circ}\right)$. a Histogram in case 1. About one-third of the particles are accelerated by about $20 \mathrm{keV}$ (energy gain by a few subpackets). Rest of the particles also gain energies less or more than $20 \mathrm{keV}$ depending on the number of the wave subpackets that each particle is entrapped by. b Histogram in case 2, which forms a distribution with the first peak at $\Delta \mathrm{K} \approx 10 \mathrm{keV}$ (energy gain by less than a single subpacket). The second peak is at $\Delta \mathrm{K} \approx 50 \mathrm{keV}$ (energy gain by a few subpackets). c Histogram in case 3, which forms a distribution with the first peak at $\Delta K \approx 10 \mathrm{keV}$ (energy gain by less than a single subpacket). The second peak is at $\Delta \mathrm{K} \approx 50 \mathrm{keV}$ (energy gain by a few subpackets). The third peak is at $\Delta \mathrm{K} \approx 100 \mathrm{keV}$ (energy gained by four or five subpackets). These distributions indicate that not only a small fraction of successful particles, but also many other particles can be accelerated with various degrees of efficiency

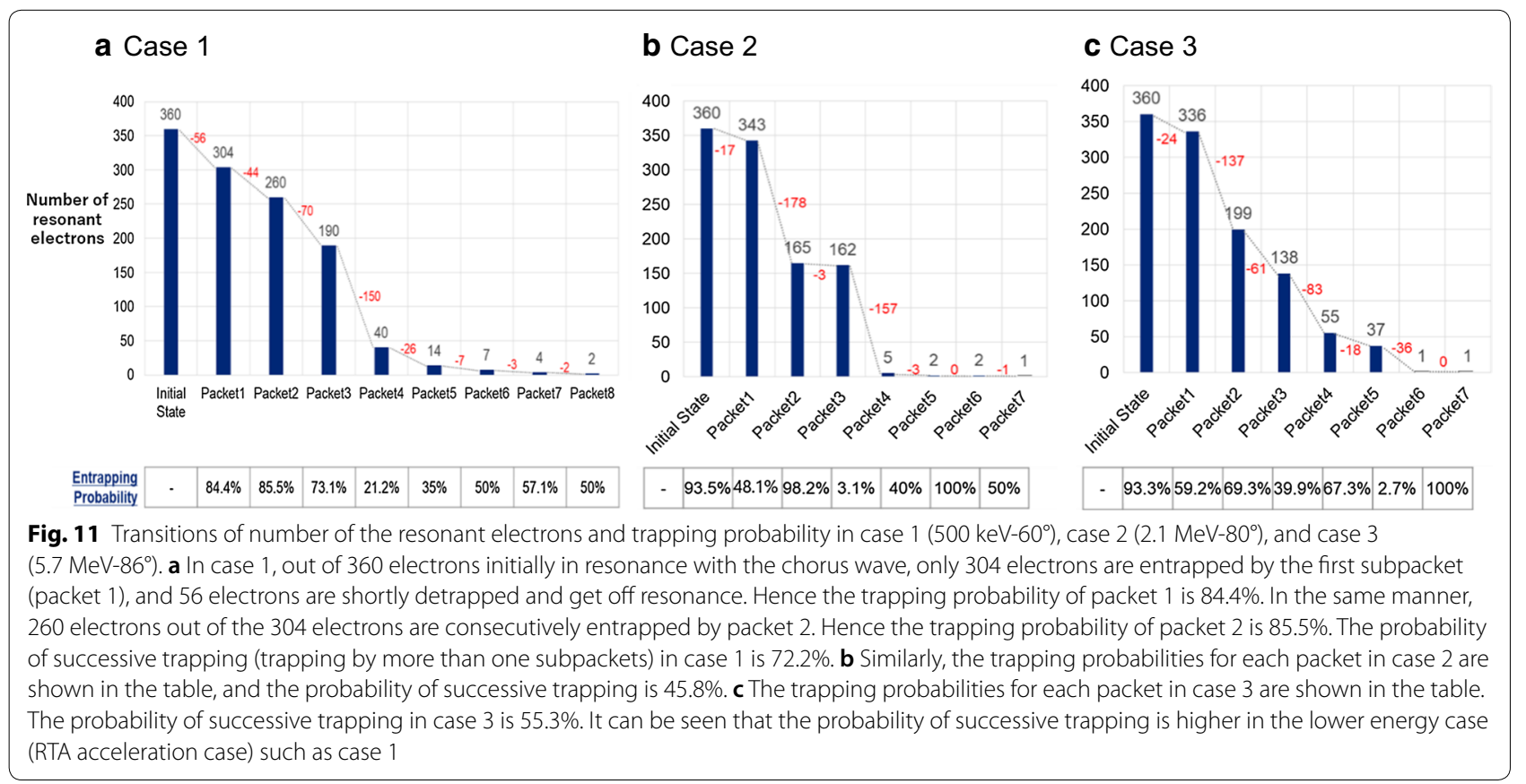

chorus element in cases 1, 2, and 3. Figure 11a shows that out of 360 electrons initially in resonance with the chorus wave, only 304 electrons are entrapped by the first subpacket (packet 1), and 56 electrons are shortly detrapped and get off resonance in case 1. Therefore, packet 1 traps resonant electrons at the probability of
$84.4 \%$. In the same manner, 260 electrons out of the 304 electrons entrapped by packet 1 are consecutively entrapped by packet 2 . Hence we obtain the trapping probability of $85.5 \%$ for packet 2 . Similarly, the trapping probabilities for other packets are obtained as shown in Fig. 11a. Since we define in this study successive 
trapping as the entrapment of electrons from the consecutive multiple (more than one) subpackets, the empirical probability of the successive trapping in case 1 becomes $72.2 \%$, calculated by 260 electrons entrapped by the first two wave subpackets out of 360 electrons. In the same method, the empirical possibilities for cases 2 and 3 are obtained as $45.8 \%$ and $55.3 \%$, respectively, as shown in Fig. 11b, c. By these results, we verify that the successive trapping occurs more frequently with lower energy electrons in the RTA acceleration (such as case 1 ), although higher energy electrons (such as cases 2 and 3) are more efficiently accelerated by URA at each trapping process as shown in Fig. 9.

\section{Summary and conclusion}

By recent observations from the Van Allen Probes (Foster et al. 2017; Omura et al. 2019), it is seen that amplitude of a single rising tone element of chorus wave consists of a large number of short wave packets, defined as multisubpacket chorus wave. Since the trapping region, where resonant electrons can be efficiently accelerated, depends on the wave amplitude, the multi-subpacket structure causes a rapid variation of the trapping region and hence affects the processes of trapping energetic electrons. In this study, we have applied a new wave model to represent the single rising tone element formed by a multisubpacket chorus wave. In the wave model, the amplitude rapidly fluctuates to form the multi-subpacket structure and the phase is randomized at the generation of each subpacket. As a result of comprehensive wave-particle simulations, an efficient trapping process defined as successive trapping has been verified. When the successive trapping occurs electrons are continuously entrapped by consecutive multiple wave subpackets, which results in efficient energy increases of electrons up to $160 \mathrm{keV}$ by the short interaction with a single rising tone chorus element.

In our simulation, acceleration by the successive trapping has been seen for electrons with various energy levels from a few hundred $\mathrm{keV}$ to $6 \mathrm{MeV}$. The successive trapping occurs during both RTA and URA accelerations. Empirically it is verified that the successive trapping occurs at a higher probability in the RTA acceleration (a larger number of electrons undergo the successive trapping in the RTA acceleration). Acceleration within each process of the successive trapping, however, is more efficient in the URA acceleration (entrapped electrons are more likely to gain higher energy from the wave in the URA acceleration). It is also verified that the interrelation between the velocity of chorus wave propagation and the electron velocity along Earth's magnetic field is a key condition in the URA process. When these two velocities are nearly the same, chorus waves cannot interact with electrons traveling in the same direction as the wave propagation, which is one of the basic conditions for the URA process. This condition occurs for electrons with an initial equatorial pitch angle of around $70^{\circ}-75^{\circ}$ in this study. Electrons with higher or lower initial equatorial pitch angles, however, interact with chorus wave by being either overtaken or catching up with chorus wave. We define the resultant accelerations as overtaken URA and catching up URA, respectively.

To conclude this study, electrons can be successfully entrapped and efficiently accelerated through interaction with a multi-subpacket rising tone chorus element both in the RTA and URA acceleration processes. It is also remarkable that electrons can be entrapped by consecutive multiple subpackets with phase discontinuities between them. Regarding the occurrence probability of the successive trapping, although the degree of energy increase is varied depending on the duration of nonlinear trapping, we find that the successive trapping can occur not only for electrons under strictly limited conditions, but also for a large number of electrons under various conditions.

\section{Abbreviations}

RTA: relativistic turning acceleration; URA: ultra-relativistic acceleration.

\section{Acknowledgements}

We thank Gerard Chanteur, Danny Summers, Satoko Nakamura, Yuko Kubota, and Yi-Kai Hsieh for their comments and discussion. Computations in the present study were performed on the KDK system of the Research Institute for Sustainable Humanosphere (RISH) at Kyoto University. This work was supported by JSPS KAKENHI Grants $15 \mathrm{H} 05815$ and $17 \mathrm{H} 06140$.

\section{Authors' contributions}

$\mathrm{RH}$ has developed simulations and conducted examinations and analysis on the theme of interaction between radiation belt electrons and subpacket chorus waves, referring to the preceding studies and simulation models. YO has provided continuing suggestion and discussion to examine the simulation results, based on plasma physics, electromagnetism, space physics, and other theoretical perspectives. Both authors read and approved the final manuscript.

\section{Funding}

This work was supported by JSPS KAKENHI Grants 15 H05815 and 17 H06140.

Availability of data and materials

Simulation codes and data can be shared on request to the authors.

Ethics approval and consent to participate

Not applicable.

\section{Consent for publication}

Not applicable.

Competing interests

The authors declare that they have no competing interests.

Received: 4 June 2019 Accepted: 12 January 2020

Published online: 19 February 2020 


\section{References}

Baker DN, Erickson PJ, Fennell JF, Foster JC, Jaynes AN, Verronen PT (2018) Space weather effects in the earth's radiation belts. Space Sci Rev. https:// doi.org/10.1007/s11214-017-0452-7

Carpenter DL (1963) Whistler evidence of a 'knee' in the magnetospheric ionization density profile. J Geophys Res 68:1675-1682

Demekhov AG, Manninen J, Santolík O, Titova EE (2017) Conjugate groundspacecraft observations of VLF chorus elements. Geophys Res Lett 44:11735-11744. https://doi.org/10.1002/2017GL076139

Dysthe KB (1971) Some studies of triggered whistler emissions. J Geophys Res 76:6915

Foster JC, Erickson PJ, Omura Y, Baker DN, Kletzing CA, Claudepierre SG (2017) Van Allen Probes observations of prompt MeV radiation belt electron acceleration in nonlinear interactions with VLF chorus. J Geophys Res Space Phys. https://doi.org/10.1002/2016JA023429

Golkowski M, Harid V, Hosseini P (2019) Review of controlled excitation of nonlinear wave-particle interactions in the magnetosphere. Front Astron Space Sci. https://doi.org/10.3389/fspas.2019.00002

Hosseini P, Gołkowski M, Turner DL (2017) Unique concurrentobservations of whistler mode hiss, chorus, and triggered emissions. J Geophys Res Space Phys 122:6271-6282. https://doi.org/10.1002/2017JA024072

Hosseini P, Gołkowski M, Harid V (2019) Remote sensing of RadiationBelt energetic electrons using lightningtriggered upper band chorus. Geophys Res Lett 46:37-47. https://doi.org/10.1029/2018GL081391

Hsieh Y-K, Omura Y (2017) Nonlinear dynamics of electrons interacting with oblique whistler mode chorus in the magnetosphere. J Geophys Res Space Phys. https://doi.org/10.1002/2016JA023255

Li W, Bortnik J, Thorne RM, Angelopoulos V (2011) Global distribution of wave amplitudes and wave normal angles of chorus waves using THEMIS wave observations. J Geophys Res 116:A12205. https://doi.org/10.1029/2011 J A017035

Matsumoto H, Omura Y (1981) Cluster and channel effect phase bunching by whistler waves in the nonuniform geomagnetic field. J Geophys Res 86:779-791

Meredith Nigel P, Thorne Richard M, Anderson Roger R (2003) Favored regions for chorus-driven electron acceleration to relativistic energies in the Earth's radiation belt. Geophys Res Lett 30(16):1871. https://doi. org/10.1029/2003GL017698

Nunn D (1974) A self-consistent theory of triggered VLF emissions. Planet Space Sci 22:349
Omura Y, Nunn D (2011) Triggering process of whistler mode chorus emissions in the magnetosphere. J Geophys Res 116:A05205. https://doi. org/10.1029/2010JA016280

Omura Y, Summers D (2006) Dynamics of high-energy electrons interacting with whistler mode chorus emissions in the magnetosphere. J Geophys Res 111:A09222. https://doi.org/10.1029/2006JA011600

Omura Y, Furuya N, Summers D (2007) Relativistic turning acceleration of resonant electrons by coherent whistler mode waves in a dipole magnetic field. J Geophys Res 112:A06236. https://doi.org/10.1029/2006JA012243

Omura Y, Katoh Y, Summers D (2008) Theory and simulation of the generation of whistler-mode chorus. J Geophys Res 113:A04223. https://doi. org/10.1029/2007JA012622

Omura Y, Hikishima M, Katoh Y, Summers D, Yagitani S (2009) Nonlinear mechanisms of lower-band and upper-band VLF chorus emissions in the magnetosphere. J Geophys Res 114:A07217. https://doi.org/10.1029/2009J A014206

Omura Y, Hsieh Y-K, Foster JC, Erickson PJ, Kletzing CA, Baker DN (2019) Cyclotron acceleration of relativistic electrons through landau resonance with obliquely propagating whistler mode chorus emissions. J Geophys Res Space Phys 124:2795-2810. https://doi.org/10.1029/2018JA026374

Santolík O, Gurnett DA (2013) Transverse dimensions of chorus in the source region. Geophys Res Lett 30(2):1031. https://doi.org/10.1029/2002GL0161 78

Santolík O, Kletzing CA, Kurth WS, Hospodarsky GB, Bounds SR (2014) Fine structure of large-amplitude chorus wave packets. Geophys Res Lett 41:293-299. https://doi.org/10.1002/2013GL058889

Shoji M, Omura Y (2013) Triggering process of electromagnetic ion cyclotron rising tone emissions in the inner magnetosphere. J Geophys Res Space Phys 118:5553-5561. https://doi.org/10.1002/jgra.50523

Summers D, Omura Y (2007) Ultra-relativistic acceleration of electrons in planetary magnetospheres. Geophys Res Lett 34:L24205. https://doi. org/10.1029/2007GL032226

Summers D, Omura Y, Miyashita Y, Lee D-H (2012) Nonlinear spatiotemporal evolution of whistler mode chorus waves in Earth's inner magnetosphere J Geophys Res 117:A09206. https://doi.org/10.1029/2012JA017842

\section{Publisher's Note}

Springer Nature remains neutral with regard to jurisdictional claims in published maps and institutional affiliations.

\section{Submit your manuscript to a SpringerOpen ${ }^{\circ}$ journal and benefit from:}

- Convenient online submission

- Rigorous peer review

- Open access: articles freely available online

- High visibility within the field

Retaining the copyright to your article

Submit your next manuscript at springeropen.com 